\title{
Using Emoticons to reduce Dependency in Machine Learning Techniques for Sentiment Classification
}

\author{
Jonathon Read \\ Department of Informatics \\ University of Sussex \\ United Kingdom \\ j.1.read@sussex.ac.uk
}

\begin{abstract}
Sentiment Classification seeks to identify a piece of text according to its author's general feeling toward their subject, be it positive or negative. Traditional machine learning techniques have been applied to this problem with reasonable success, but they have been shown to work well only when there is a good match between the training and test data with respect to topic. This paper demonstrates that match with respect to domain and time is also important, and presents preliminary experiments with training data labeled with emoticons, which has the potential of being independent of domain, topic and time.
\end{abstract}

\section{Introduction}

Recent years have seen an increasing amount of research effort expended in the area of understanding sentiment in textual resources. A sub-topic of this research is that of Sentiment Classification. That is, given a problem text, can computational methods determine if the text is generally positive or generally negative? Several diverse applications exist for this potential technology, ranging from the automatic filtering of abusive messages (Spertus, 1997) to an in-depth analysis of market trends and consumer opinions (Dave et al., 2003). This is a complex and challenging task for a computer to achieve - consider the difficulties involved in instructing a computer to recognise sarcasm, for example.
Previous work has shown that traditional text classification approaches can be quite effective when applied to the sentiment analysis problem. Models such as Naïve Bayes (NB), Maximum Entropy (ME) and Support Vector Machines (SVM) can determine the sentiment of texts. Pang et al. (2002) used a bagof-features framework (based on unigrams and bigrams) to train these models from a corpus of movie reviews labelled as positive or negative. The best accuracy achieved was $82.9 \%$, using an SVM trained on unigram features. A later study (Pang and Lee, 2004) found that performance increased to $87.2 \%$ when considering only those portions of the text deemed to be subjective.

However, Engström (2004) showed that the bagof-features approach is topic-dependent. A classifier trained on movie reviews is unlikely to perform as well on (for example) reviews of automobiles. Turney (2002) noted that the unigram unpredictable might have a positive sentiment in a movie review (e.g. unpredictable plot), but could be negative in the review of an automobile (e.g. unpredictable steering). In this paper, we demonstrate how the models are also domain-dependent - how a classifier trained on product reviews is not effective when evaluating the sentiment of newswire articles, for example. Furthermore, we show how the models are temporally-dependent - how classifiers are biased by the trends of sentiment apparent during the time-period represented by the training data.

We propose a novel source of training data based on the language used in conjunction with emoticons in Usenet newsgroups. Training a classifier using this data provides a breadth of features that, while it 


\begin{tabular}{ll|rrr}
\hline & & $\begin{array}{r}\text { Testing } \\
\text { FIN }\end{array}$ & M\&A & MIX \\
Training & & & & \\
\hline NB & FIN & $\mathbf{8 0 . 3}$ & $\mathbf{7 5 . 5}$ & 74.0 \\
& M\&A & 77.5 & 75.3 & 75.8 \\
& MIX & 70.7 & 62.9 & $\mathbf{8 4 . 6}$ \\
& & & & \\
SVM & FIN & $\mathbf{7 8 . 8}$ & 72.7 & 68.9 \\
& M\&A & 74.5 & $\mathbf{7 5 . 5}$ & 75.5 \\
& MIX & 72.0 & 68.9 & $\mathbf{8 1 . 1}$ \\
\hline
\end{tabular}

Figure 1: Topic dependency in sentiment classification. Accuracies, in percent. Best performance on a test set for each model is highlighted in bold.

does not perform to the state-of-the-art, could function independent of domain, topic and time.

\section{Dependencies in Sentiment Classification}

\subsection{Experimental Setup}

In this section, we describe experiments we have carried out to determine the influence of domain, topic and time on machine learning based sentiment classification. The experiments use our own implementation of a Naïve Bayes classifier and Joachim's (1999) $S V M^{\text {light }}$ implementation of a Support Vector Machine classifier. The models were trained using unigram features, accounting for the presence of feature types in a document, rather than the frequency, as Pang et al. (2002) found that this is the most effective strategy for sentiment classification.

When training and testing on the same set, the mean accuracy is determined using three-fold crossvalidation. In each case, we use a paired-sample t-test over the set of test documents to determine whether the results produced by one classifier are statistically significantly better than those from another, at a confidence interval of at least $95 \%$.

\subsection{Topic Dependency}

Engström (2004) demonstrated how machinelearning techniques for sentiment classification can be topic dependent. However, that study focused on a three-way classification (positive, negative and neutral). In this paper, for uniformity across different data sets, we focus on only positive and negative sentiment. This experiment also provides an opportunity to evaluate the Naïve Bayes classifier as the previous work used SVMs.

We use subsets of a Newswire dataset (kindly pro-

\begin{tabular}{ll|rr}
\hline & & $\begin{array}{r}\text { Testing } \\
\text { Newswire }\end{array}$ & Polarity 1.0 \\
Training & & & \\
\hline NB & Newswire & $\mathbf{7 8 . 2}$ & 57.6 \\
& Polarity 1.0 & 53.2 & $\mathbf{7 8 . 9}$ \\
\multirow{2}{*}{ SVM } & Newswire & $\mathbf{7 8 . 2}$ & 63.2 \\
& Polarity 1.0 & 63.6 & $\mathbf{8 1 . 5}$ \\
\hline
\end{tabular}

Figure 2: Domain dependency in sentiment classification. Accuracies, in percent. Best performance on a test set for each model is highlighted in bold.

vided by Roy Lipski of Infonic Ltd.) that relate to the topics of Finance (FIN), Mergers and Aquisitions (M\&A) and a mixture of both topics (MIX). Each subset contains further subsets of articles of positive and negative sentiment (selected by independent trained annotators), each containing 100 stories. We trained a model on a dataset relating to one topic and tested that model using the other topics. Figure 1 shows the results of this experiment.

The tendency seems to be that performance in a given topic is best if the training data is from the same topic. For example, the Finance-trained SVM classifier achieved an accuracy of $78.8 \%$ against articles from Finance, but only $72.7 \%$ when predicting the sentiment of articles from M\&A. However, statistical testing showed that the results are not significantly different when training on one topic and testing on another. It is interesting to note, though, that providing a dataset of mixed topics (the sub-corpus MIX) does not necessarily reduce topic dependency. Indeed, the performance of the classifiers suffers a great deal when training on mixed data (confidence interval 95\%).

\subsection{Domain Dependency}

We conducted an experiment to compare the accuracy when training a classifier on one domain (newswire articles or movie reviews from the Polarity 1.0 dataset used by Pang et al. (2002)) and testing on the other domain. In Figure 2, we see a clear indication that models trained on one domain do not perform as well on another domain. All differences are significant at a confidence interval of $99.9 \%$.

\subsection{Temporal Dependency}

To investigate the effect of time on sentiment classification, we constructed a new set of movie re- 


\begin{tabular}{ll|rr}
\hline & & $\begin{array}{r}\text { Testing } \\
\text { Polarity 1.0 }\end{array}$ & Polarity 2004 \\
Training & & & \\
\hline NB & Polarity 1.0 & $\mathbf{7 8 . 9}$ & 71.8 \\
& Polarity 2004 & 63.2 & $\mathbf{7 6 . 5}$ \\
\multirow{2}{*}{ SVM } & Polarity 1.0 & $\mathbf{8 1 . 5}$ & 77.5 \\
& Polarity 2004 & 76.5 & $\mathbf{8 0 . 8}$ \\
\hline
\end{tabular}

Figure 3: Temporal dependency in sentiment classification. Accuracies, in percent. Best performance on a test set for each model is highlighted in bold.

views, following the same approach used by Pang et al. (2002) when they created the Polarity 1.0 dataset. The data source was the Internet Movie Review Database $\operatorname{archive}^{1}$ of movie reviews. The reviews were categorised as positive or negative using automatically extracted ratings. A review was ignored if it was not written in 2003 or 2004 (ensuring that the review was written after any in the Polarity 1.0 dataset). This procedure yielded a corpus of 716 negative and 2,669 positive reviews. To create the Polarity $2004^{2}$ dataset we randomly selected 700 negative reviews and 700 positive reviews, matching the size and distribution of the Polarity 1.0 dataset.

The next experiment evaluated the performance of the models first against movie reviews from the same time-period as the training set and then against reviews from the other time-period. Figure 3 shows the resulting accuracies.

These results show that while the models perform well on reviews from the same time-period as the training set, they are not so effective on reviews from other time-periods (confidence interval 95\%). It is also apparent that the Polarity 2004 dataset performs worse than the Polarity 1.0 dataset (confidence interval 99.9\%). A possible reason for this is that Polarity 2004 data is from a much smaller time-period than that represented by Polarity 1.0.

\section{Sentiment Classification using Emoticons}

One way of overcoming the domain, topic and time problems we have demonstrated above would be to find a source of much larger and diverse amounts of general text, annotated for sentiment. Users of

\footnotetext{
${ }^{1}$ http: //reviews.imdb.com/Reviews /

${ }^{2}$ The new datasets described in this paper are available at http://www.sussex.ac.uk/Users/jlr24/data
}

\begin{tabular}{c|l|r}
\hline Glyph & Meaning & Frequency \\
\hline$:-)$ & smile & 3.8739 \\
;-) & wink & 2.4350 \\
:-( & frown & 0.4961 \\
:-D & wide grin & 0.1838 \\
:-P & tongue sticking out & 0.1357 \\
:-O & surprise & 0.0171 \\
:-| & disappointed & 0.0146 \\
$: '($ & crying & 0.0093 \\
$:-\mathrm{S}$ & confused & 0.0075 \\
$:-@$ & angry & 0.0038 \\
$:-\$$ & embarrassed & 0.0007 \\
\hline
\end{tabular}

Figure 4: Examples of emoticons and the frequency of usage observed in Usenet articles, in percent. For example, $2.435 \%$ of downloaded Usenet articles contained a wink emoticon.

electronic methods of communication have developed visual cues that are associated with emotional states in an attempt to state the emotion that their text represents. These have become known as smileys or emoticons and are glyphs constructed using the characters available on a standard keyboard, representing a facial expression of emotion - see Figure 4 for some examples. When the author of an electronic communication uses an emoticon, they are effectively marking up their own text with an emotional state. This marked-up text can be used to train a sentiment classifier if we assume that a smile indicates generally positive text and a frown indicates generally negative text.

\subsection{Emoticon Corpus Construction}

We collected a corpus of text marked-up with emoticons by downloading Usenet newsgroups and saving an article if it contained an emoticon listed in Figure 4. This process resulted in 766,730 articles being stored, from 10,682,455 messages in 49,759 newsgroups inspected. Figure 4 also lists the percentage of documents containing each emoticon type, as observed in the Usenet newsgroups.

We automatically extracted the paragraph(s) containing the emoticon of interest (a smile or a frown) from each message and removed any superfluous formatting characters (such as those used to indicate article quotations in message threads). In order to prevent quoted text from being considered more than once, any paragraph that began with exactly the same thirty characters as a previously observed paragraph was disregarded. Finally, we used the classifier developed by Cavnar and Trenkle (1994) to filter 


\begin{tabular}{l|rrr}
\hline & Finance & M\&A & Mixed \\
\hline NB & $46.0 \pm 2.1$ & $55.8 \pm 3.8$ & $49.0 \pm 1.6$ \\
SVM & $50.3 \pm 1.7$ & $57.8 \pm 6.5$ & $55.5 \pm 2.7$ \\
\hline
\end{tabular}

Figure 5: Performance of Emoticon-trained classifier across topics. Mean accuracies with standard deviation, in percent.

\begin{tabular}{l|rr}
\hline & Newswire & Polarity 1.0 \\
\hline NB & $50.3 \pm 2.2$ & $56.8 \pm 1.8$ \\
SVM & $54.4 \pm 2.8$ & $54.0 \pm 0.8$ \\
\hline
\end{tabular}

Figure 6: Performance of Emoticon-trained classifiers across domains. Mean accuracies with standard deviation, in percent.

out any paragraphs of non-English text. This process yielded a corpus of 13,000 article extracts containing frown emoticons. As investigating skew between positive and negative distributions is outside the scope of this work, we also extracted 13,000 article extracts containing smile emoticons. The dataset is referred to throughout this paper as Emoticons and contains 748,685 words.

\subsection{Emoticon-trained Sentiment Classification}

This section describes how the Emoticons corpus ${ }^{3}$ was optimised for use as sentiment classification training data. 2,000 articles containing smiles and 2,000 articles containing frowns were held-out as optimising test data. We took increasing amounts of articles from the remaining dataset (from 2,000 to 22,000 in increments of 1,000 , an equal number being taken from the positive and negative sets) as optimising training data. For each set of training data we extracted a context of an increasing number of tokens (from 10 to 1,000 in increments of 10) both before and in a window ${ }^{4}$ around the smile or frown emoticon. The models were trained using this extracted context and tested on the held-out dataset.

The optimisation process revealed that the bestperforming settings for the Naïve Bayes classifier was a window context of 130 tokens taken from the largest training set of 22,000 articles. Similarly, the best performance for the SVM classifier was found using a window context of 150 tokens taken from

\footnotetext{
${ }^{3}$ Note that in these experiments the emoticons are used as anchors from which context is extracted, but are removed from texts before they are used as training or test data.

${ }^{4}$ Context taken after an emoticon was also investigated, but was found to be inferior. This is because approximately twothirds of article extracts end in an emoticon so when using aftercontext few features are extracted.
}

\begin{tabular}{l|rr}
\hline & Polarity 1.0 & Polarity 2004 \\
\hline NB & $56.8 \pm 1.8$ & $56.7 \pm 2.2$ \\
SVM & $54.0 \pm 0.8$ & $57.8 \pm 1.8$
\end{tabular}

Figure 7: Performance of Emoticon-trained classifier across time-periods. Mean accuracies with standard deviation, in percent.

20,000 articles.

The classifiers' performance in predicting the smiles and frowns of article extracts was verified using these optimised parameters and ten-fold crossvalidation. The mean accuracy of the Naïve Bayes classifier was $61.5 \%$, while the SVM classifier was $70.1 \%$.

Using these same classifiers to predict the sentiment of movie reviews in Polarity 1.0 resulted in accuracies of $59.1 \%$ (Naïve Bayes) and $52.1 \%$ (SVM).

We repeated the optimisation process using a held-out set of 100 positive and 100 negative reviews from the Polarity 1.0 dataset, as it is possible that this test needs different parameter settings. This revealed an optimum context of a window of 50 tokens taken from a training set of 21,000 articles for the Naïve Bayes classifier. Interestingly, the optimum context for the SVM classifier appeared to be a window of only 20 tokens taken from a mere 2,000 training examples. This is clearly an anomaly, as these parameters resulted in an accuracy of $48.9 \%$ when testing against the reserved reviews of Polarity 1.0. We attribute this to the presence of noise, both in the training set and in the held-out set, and discuss this below (Section 4.2). The second-best parameters according to the optimisation process were a context of 510 tokens taken before an emoticon, from a training set of 20,000 examples.

We used these optimised parameters to evaluate the sentiments of texts in the test sets used to evaluate dependency in Section 2. Figures 5, 6 and 7 show the final, optimised results across topics, domains and time-periods respectively. These tables report the average accuracies over three folds, with the standard deviation as a measure of error.

\section{Discussion}

The emoticon-trained classifiers perform well (up to $70 \%$ accuracy) when predicting the sentiment of article extracts from the Emoticons dataset, which is encouraging when one considers the high level of 


\begin{tabular}{ll|r}
\hline Training & Testing & Coverage \\
\hline Polarity 1.0 & Polarity 1.0 & 69.8 \\
& (three-fold cross-validation) & \\
Emoticons & FIN & 54.9 \\
& M\&A & 58.1 \\
& MIX & 60.2 \\
& Newswire & 46.1 \\
& Polarity 1.0 & 41.1 \\
& Polarity 2004 & 42.6 \\
\hline
\end{tabular}

Figure 8: Coverage of classifiers, in percent.

noise that is likely to be present in the dataset.

However, they perform only a little better than one would expect by chance when classifying movie reviews, and are not effective in predicting the sentiment of newswire articles. This is perhaps due to the nature of the datasets - one would expect language to be informal in movie reviews, and even more so in Usenet articles. In contrast, language in newswire articles is far more formal. We might therefore infer a further type of dependence in sentiment classification, that of language-style dependency.

Also, note that neither machine-learning model consistently out-performs the other. We speculate that this, and the generally mediocre performance of the classifiers, is due (at least) to two factors; poor coverage of the features found in the test domains and a high level of noise found in Usenet article extracts. We investigate these factors below.

\subsection{Coverage}

Figure 8 shows the coverage of the Emoticon-trained classifiers on the various test sets. In these experiments, we are interested in the coverage in terms of unique token types rather than the frequency of features, as this more closely reflects the training of the models (see Section 2.1). The mean coverage of the Polarity 1.0 dataset during three-fold crossvalidation is also listed as an example of the coverage one would expect from a better-performing sentiment classifier. The Emoticon-trained classifier has much worse coverage in the test sets.

We analysed the change in coverage of the Emoticon-trained classifiers on the Polarity 1.0 dataset. We found that the coverage continued to improve as more training data was provided; the coverage of unique token types was improving by about $0.6 \%$ per 1,000 training examples when the Emoti-

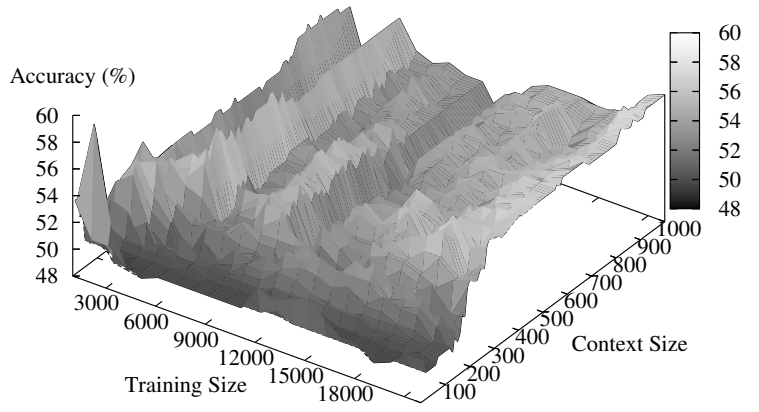

Figure 9: Change in Performance of the SVM Classifier on held-out reviews from Polarity 1.0, varying training set size and window context size. The datapoints represent 2,200 experiments in total.

cons dataset was exhausted.

It appears possible that more training data will improve the performance of the Emoticon-trained classifiers by increasing the coverage. Potential sources for this include online bulletin boards, chat forums, and further newsgroup data from Usenet and Google Groups ${ }^{5}$. Future work will utilise these sources to collect more examples of emoticon use and analyse any improvement in coverage and accuracy.

\subsection{Noise in Usenet Article Extracts}

The article extracts collected in the Emoticons dataset may be noisy with respect to sentiment. The SVM classifier seems particularly affected by this noise. Figure 9 depicts the change in performance of the SVM classifier when varying the training set size and size of context extracted. There are significant spikes apparent for the training sizes of 2,000, 3,000 and 6,000 article extracts (as noted in Section 3.2), where the accuracy suddenly increases for the training set size, then quickly decreases for the next set size. This implies that the classifier is discovering features that are useful in classifying the heldout set, but the addition of more, noisy, texts soon makes the information redundant.

Some examples of noise taken from the Emoticons dataset are: mixed sentiment, e.g.

\footnotetext{
${ }^{5}$ http://groups.google.com
} 
"Sorry about venting my frustration here but I just lost it. :-( Happy thanks giving everybody :-)",

sarcasm, e.g.

"Thank you so much, that's really encouraging :-(",

and spelling mistakes, e.g.

"The movies where for me a major desapointment :-(".

In future work we will investigate ways to remove noisy data from the Emoticons dataset.

\section{Conclusions and Future Work}

This paper has demonstrated that dependency in sentiment classification can take the form of domain, topic, temporal and language style. One might suppose that dependency is occurring because classifiers are learning the semantic sentiment of texts rather than the general sentiment of language used. That is, the classifiers could be learning authors' sentiment towards named entities (e.g. actors, directors, companies, etc.). However, this does not seem to be the case. In a small experiment, we part-ofspeech tagged the Polarity 2004 dataset and automatically replaced proper nouns with placeholders. Retraining on this modified text did not significantly affect performance.

But it may be that something more subtle is happening. Possibly, the classifiers are learning the words associated with the semantic sentiment of entities. For example, suppose that there has been a well-received movie about mountaineering. During this movie, there is a particularly stirring scene involving an ice-axe and most of the reviewers mention this scene. During training, the word 'ice-axe' would become associated with a positive sentiment, whereas one would suppose that this word does not in general express any kind of sentiment.

In future work we will perform further tests to determine the nature of dependency in machine learning techniques for sentiment classification. One way of evaluating the 'ice-axe' effect could be to build a 'pseudo-ontology' of the movie reviews - a map of the sentiment-bearing relations that would enable the analysis of the dependencies created by the training process. Other extensions of this work are to collect more text marked-up with emoticons, and to experiment with techniques to automatically remove noisy examples from the training data.

\section{Acknowledgements}

This research was funded by a UK EPSRC studentship. I am very grateful to Thorsten Joachims, Roy Lipski, Bo Pang and John Trenkle for kindly making their data or software available, and to the anonymous reviewers for their constructive comments. Thanks also to Nick Jacobi for his discussion of the 'ice-axe' effect. Special thanks to my supervisor, John Carroll, for his continued advice and encouragement.

\section{References}

W. B. Cavnar and J. M. Trenkle. 1994. N-Gram-Based Text Categorization. In Proceedings of the Third Annual Symposium on Document Analysis and Information Retrieval, pages 161-175, Las Vegas, Nevada.

Kushal Dave, Steve Lawrence, and David M. Pennock. 2003. Mining the Peanut Gallery: Opinion Extraction and Semantic Classification of Product Reviews. In Proceedings of the International World Wide Web Conference, Budapest, Hungary.

Charlotta Engström. 2004. Topic Dependence in Sentiment Classification. Master's thesis, University of Cambridge, July.

T. Joachims. 1999. Making large-Scale SVM Learning Practical. In B. Schölkopf, C. Burges, and A. Smola, editors, Advances in Kernel Methods - Support Vector Learning. MIT Press.

Bo Pang and Lillian Lee. 2004. A Sentimental Education: Sentiment Analysis Using Subjectivity Summarization Based on Minimum Cuts. In Proceedings of the 42nd Annual Meeting of the Association for Computational Linguistics, Barcelona, Spain.

Bo Pang, Lillian Lee, and Shivakumar Vaithyanathan. 2002. Thumbs up? Sentiment Classification using Machine Learning Techniques. In Proceedings of the 2002 Conference on Empirical Methods in Natural Language Processing, University of Pennsylvania.

Ellen Spertus. 1997. Smokey: Automatic Recognition of Hostile Messages. In Proceedings of the Innovative Applications of Artificial Intelligence.

Peter D. Turney. 2002. Thumbs up or thumbs down? Semantic orientation applied to unsupervised classification of reviews. In Proceedings of the 40th Annual Meeting of the Association for Computational Linguistics (ACL'02), pages 417-424, Philadelphia, Pennsylvania. 